\title{
Real-Time Scalable Visual Tracking via Quadrangle Kernelized Correlation Filters
}

\author{
Guiguang Ding, Wenshuo Chen, Sicheng Zhao, Jungong Han, Qiaoyan Liu
}

\begin{abstract}
Correlation filter (CF) has been widely used in tracking tasks due to its simplicity and high efficiency. However, conventional $\mathrm{CF}$ based trackers fail to handle the scale variation occurs when the targeted object is moving, which is one of the most notable unsolved problems of visual object tracking. In this paper, we propose a scalable visual tracking algorithm based on kernelized correlation filters, referred to as Quadrangle Kernelized Correlation Filters (QKCF). Unlike existing complicated scalable trackers that either perform the correlation filtering operation multiple times or extract many candidate windows at various scales, our tracker intends to estimate the scale of the object based on the positions of its four corners, which can be detected using a new Gaussian training output matrix within one filtering process. After obtaining four peak values corresponding to the four corners, we measure the detection confidence of each part response by evaluating its spatial and temporal smoothness. On top of it, a weighted Bayesian inference framework is employed to estimate the final location and size of the bounding box from the response matrix, where the weights are synchronized with the calculated detection likelihoods. Experiments are performed on the OTB-100 dataset and 16 benchmark sequences with significant scale variations. The results demonstrate the superiority of the proposed method in terms of both effectiveness and robustness, compared to the state-of-the-arts.
\end{abstract}

Index Terms-Image emotion, probability distribution, valence-arousal, Gaussian mixture model, shared sparse regression, multi-task learning

\section{INTRODUCTION}

With the rapid development of digital multimedia technology, the total amount of video data, such as user-generated videos and surveillance videos, has been explosively increasing, which poses a great challenge to Internet inspection and security monitoring. As an important technique in computer vision, visual object tracking [1], [2] aims to estimate the location of a visual target at each frame of an image sequence and plays an important role in intelligent transportation systems [3], [4]. Due to the complexity of real-world scenes, such as illumination variation, partial occlusion, background

Copyright (c) 2013 IEEE. Personal use of this material is permitted. However, permission to use this material for any other purposes must be obtained from the IEEE by sending a request to pubs-permissions@ieee.org.

Manuscript received March 30, 2017. This work was supported by the National Natural Science Foundation of China (No. 61571269, 61671267, 61701273), China Postdoctoral Science Foundation (No. 2017M610897), and the Royal Society Newton Mobility Grant (IE150997). Corresponding authors: Jungong Han; Sicheng Zhao

G. Ding, W. Chen, S. Zhao and Q. Liu are with the School of Software, Tsinghua University, Beijing 100084, China (e-mail: dinggg@tsinghua.edu.cn; sabrinachen321@gmail.com; schzhao@gmail.com; liuqy16@mails.tsinghua.edu.cn).

J. Han is with School of Computing and Communications, Lancaster University, Lancaster, LA1 4YW, UK (e-mail: jungong.han@lancaster.ac.uk).

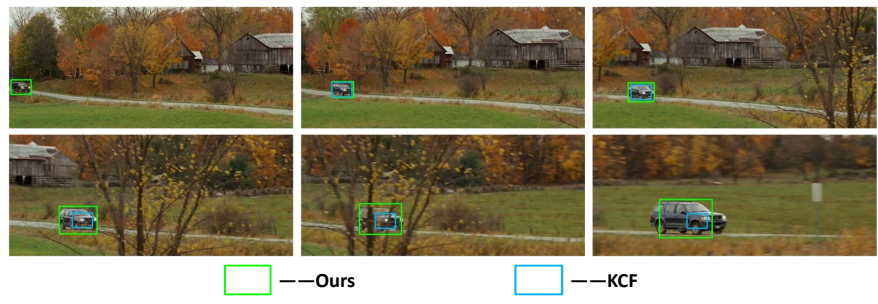

Fig. 1. Comparisons of the proposed QKCF with KCF tracker [5] in the challenging situation of scale variation on the CarScale sequence [6]. While preserving the high tracking speed of KCF, our tracker can well handle the scale variation problem by a Gaussian training output matrix with four peak values in one filter.

clutter, motion blur and scale variation, visual tracking remains a challenging task.

In recent years, tracking based on correlation filter (CF) [5], [7]-[10] has shown to accurately provide the object location in real-time. The underlying principle behind these approaches lies in the exploration of the circulant matrix structure in tracking scenes, which can be efficiently computed using convolution theorem based on a fast Fourier transform (FFT). Bolme et al. [7] presented a pioneering research that uses CF for visual tracking on gray-scale images to learn a minimum output sum of squared error (MOSSE). The tracking speed of MOSSE tracker reaches several hundreds frames per second $(\mathrm{f} / \mathrm{s})$, i.e. $669 \mathrm{f} / \mathrm{s}$ on low resolution videos. This is deemed to be a remarkable improvement, in contrast to the existing trackers like Struck [11], TLD [12] and MIL [13] and ORIA [14], where the first three can hardly reach $30 \mathrm{f} / \mathrm{s}$ while the last one only gets $9 \mathrm{f} / \mathrm{s}$ in the equivalent circumstance. There are a lot of improved versions of MOSSE, such as CSK [8], CAT [9], spatio-temporal context (STC) [10] and KCF [5], in which the CSK tracker [8] that extends the CF in a kernel space, turns out to be the most outstanding one in terms of the tracking speed [6]. Late on, replacing the gray-scale representation with the HOG features [15] allows the KCF tracker [5] to achieve a good trade-off between tracking speed and tracking accuracy.

In spite of satisfactory performance achieved in the simple environment, all the above mentioned CF trackers suffer from the problem that the scale of targeted object changes dramatically over time, which occurs frequently in the real-world scenarios, as illustrated in Figure 1. To solve this problem, some recent visual trackers adopt either part based multiple filters [16] or multi-scale spatial pyramid [17], [18], in which the optimal scale is estimated by repeatedly applying the correlation filtering to the candidate regions sampled from different image scales. Despite its great success in detecting 
the object-scale variation for most practical situations, the computational efficiency is dramatically degraded due to the multiple filtering operations carried out in one single frame.

In this paper, we aim to solve the scale variation problem in a single-frame-single-filter framework. Based on the intrinsic boundary extension property of CF trackers, we introduce a quadrangle Gaussian training label matrix to fully explore the boundary information of the target object. By improving the training and tracking algorithms of $\mathrm{KCF}$ [5], we propose Quadrangle Kernelized Correlation Filters (QKCF), which can simultaneously obtain the location and scale of the target object in only one filter.

The CF trackers usually train a model based on the labeled bounding box of the target object in the first frame and then determine the offset location of the target object in subsequentl frames by $\mathrm{CF}$ operations, which is called target translation detection. Without taking the scale variation into consideration, the traditional $\mathrm{KCF}$ tracker [5] only requires obtaining the centre point of the target object, which can be easily accomplished by selecting the offset with the maximum response. However, the proposed quadrangle Gaussian training label matrix relies on the boundary information instead of the centre point. Therefore, a straightforward duplication of maximum response will no longer work in this context.

To tackle this problem, a weighted Bayesian inference framework is proposed to estimate the location and size of the bounding box from the four-peak-value response matrix. More specifically, it adaptively measures each part response both spatially and temporally, and then makes a biased combination of all confidence maps based on the response weights. In practice, some parts of the object may be occluded or undergo illumination changes, thereby producing unreliable information. In our formulation, we adaptively adjust the weights such that the information from unreliable parts contributes less during the combination. We conduct extensive experiments on the OTB-100 dataset and 16 benchmark sequences with significant scale variations. The experimental results demonstrate the superiority of the proposed method in terms of tracking accuracy, efficiency and robustness, compared to the state-ofthe-art tracking methods.

The rest of this paper is organized as follows. Section II reviews related work on visual tracking. The detailed algorithms, i.e., the quadrangle kernelized correlation filter and weighted Bayesian inference are described in Section III and Section IV respectively. Experimental evaluation and analysis are presented in Section VI followed by conclusion and future work in Section VII.

\section{RELATED WORKS}

Visual tracking has been extensively studied and numerous trackers [1], [2], [6], [19] have been proposed in the past decade. In this section, we briefly discuss the methods closely related to our work, which are (i) CF based trackers and (ii) scalable visual trackers.

Correlation filter based trackers: As a popular measurement of the correlation or similarity between two signals, correlation filter (CF) has been widely used in various applications, such as eye localization and object detection [20]. Since the $\mathrm{CF}$ operator can be easily transferred into the Fourier domain, on which the correlation can be efficiently computed via FFT, CF-based trackers have been widely used in real-time applications [5], [7]-[10]. Having initialized a small window centered on the object in the first frame, the target is tracked by correlating the filter over a larger searching window in next frame and the new location of the target is specified by looking at the maximum correlation response. This new location is in turn used to updated the filter.

MOSSE [7] is the first CF based tracker, which directly takes the gray values as visual features and detects the target object via a linear CF classifier. The tracking speed of MOSSE can reach several hundreds frames per second with the aid of $\mathrm{CF}$. Heriques et al. proposed extending $\mathrm{CF}$ to a kernel space by the CSK method [8], which is built upon the illumination intensity features. Both KCF tracker [5] and CAT tracker [9] are the extended multi-channel versions of CSK, where the former one adopted the geometry and illumination invariant HOG features while the latter replaced the original gray scale representation with the color attributes. Zhang et al. [10] changed the Gaussian function in MOSSE to a Bayesian framework such that the contextual information can be incorporated into the filter learning. This STC track can model the scale change to limited extent based on consecutive correlation responses. To sum up, all the above methods are restricted to only estimating the target translation so that they generally yield a poor performance in complex scenarios where significant scale changes occur.

Scalable visual trackers: The phenomenon of scale change is a common yet challenging problem in computer vision [21]. In the framework of discriminative model based trackingby-detection [12], [22], how to automatically estimate the scale of target object has kept researchers busy for a long while. Among the available proposals, part based detection and multi-scale spatial pyramid seem excellent. DSST [18] and LCT [17] are two typical trackers of multi-scale spatial pyramid under the CSK framework. DSST tracker [18] firstly used the MOSSE algorithm to find the maximum response of the object indicating the object translation. Next, a separate scale detection model is trained to search the optimal scale in the multi-scale spatial pyramid. By learning discriminative correlation filters, the scale in LCT [17] is estimated by searching the target appearance pyramid exhaustively while the translation is estimated by modeling the temporal context correlation. Though performed pretty well on video sequences with significant scale variation, DSST [18] and LCT [17] both greatly reduce the tracking efficiency of a traditional CSK due to the heavy computation load spent on training and detecting object across the multi-scale images. Alternatively, PBT method [16] divided the target object into multiple parts and learned $\mathrm{CF}$ for each part separately. A Bayesian inference framework was adopted to estimate the object's new location and scale by combining the tracking results of all parts. Experiments showed that PBT can well handle the foreground occlusion and scale variation. But again, multi-scale filtering is operated at each localization step, which dramatically reduces the tracking speed. 
Deep trackers: Very recently, owing to the powerful feature learning capability of $\mathrm{CNN}$, deep networks have been introduced into visual tracking. Due to lacks of training data, CNNs are usually pretrained on a large-scale dataset for image classification [23] [24] [25] [26] to learn a generic relationship between object motion and appearance in either an on-line or off-line manner. Late on, [27] [28] [29] propose to train the CNNs on a set of annotated video sequences instead of still images, and the obtained results showed that the CNNs trained on video sequences are more robust to the environmental variations. In contrast to these deep trackers that reply on a large training set, our algorithm aims to tracking scaling objects based on fast $\mathrm{CF}$ technique with no need for an intensive training procedure.

It is noted that there are some other tracking methodologies related to our research, including tracking-by-detection [12], [22], ensemble tracking [30]-[33], contour tracking [34], [35], hash tracking [36], multi-cue tracking [37] and multi-object tracking [38]-[42]. Tracking-by-detection algorithms [12], [22] base their trackers on the object detection results. Differently, ensemble tracking [30]-[33] combines a set of weak classifiers, which are trained online to distinguish the object from background, into a strong classifier to label pixels in the next frame. Contour tracking [34], [35] aims to track the fine-grained contours instead of simple rectangles, which is often used for non-rigid objects. Hash tracking maps the high-dimensional data to a compact binary code, solving the scale and dimension increasing problem with a constant time. Multi-cue tracking [37] jointly employs different cues about the object for the accurate localization of target object in extreme conditions, while multi-object tracking [38]-[42] tracks multiple objects simultaneously. The proposed QKCF can be deemed as one kind of tracking-by-detection trackers but with high accuracy and fast speed. Distinct from ensemble tracking, contour tracking, hash tracking, multi-cue tracking and multi-object tracking, the proposed method is a nonensemble, non-contour, single-cue and single-object tracker.

\section{Quadrangle Kernelized Correlation Filter}

Basically, we aim to build a real-time tracking system that is robust to scale variations, and meanwhile, avoid using timeconsuming multi-sampling strategy or multi-scale space pyramid. Due to the high efficiency and competitive performance of KCF [5], we base our method on the KCF tracker. The key idea is to employ a Gaussian training output matrix with four peak values to implement multiple edges tracking in one filter operation. Furthermore, a weighted Bayesian inference framework is proposed to deduce the location and size of the bounding box simultaneously. The flowchart despicting the whole process is shown in Figure 2

\section{A. The KCF Tracker}

In this section, we briefly introduce the KCF tracker. More details can be referred to [5]. Using the ridge regression as a filtering model, KCF aims to find a function $f(\mathbf{z})=\mathbf{w}^{T} \mathbf{z}$ that minimizes the squared error over samples $\mathbf{x}_{i}$ and their

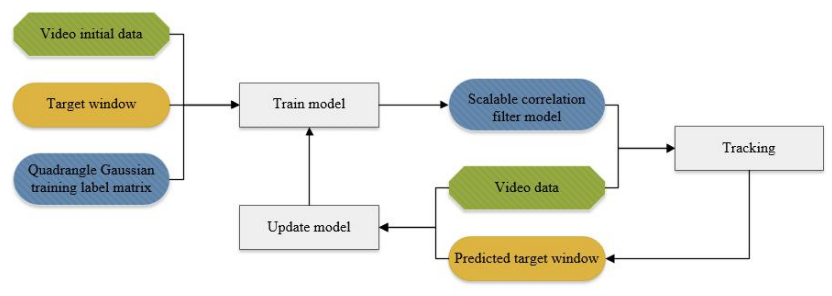

Fig. 2. Flowchart of QKCF process. At the first frame, initial frame data and the groundtruth are used to train the first correlation filter model. After predicting the target window of the next frame by the model, we use the frame data and the target window to update the model, and then predict the next frame again.

regression targets $y_{i}$,

$$
\min _{\mathbf{w}} \sum_{i}\left(f\left(\mathbf{x}_{i}\right)-y_{i}\right)^{2}+\lambda\|\mathbf{w}\|^{2}
$$

where $\mathbf{w}$ is kernelized filtering template and $\lambda$ is a regularization parameter that controls overfitting. Suppose the kernel function is $\kappa\left(\mathbf{x}, \mathbf{x}^{\prime}\right)=\left\langle\varphi(\mathbf{x}), \varphi\left(\mathbf{x}^{\prime}\right)\right\rangle$. After mapping the inputs of a linear problem to a nonlinear feature-space $\varphi(\mathbf{x})$, the closed form solution of ridge regression is

$$
\mathbf{w}=\sum_{i} \alpha_{i} \varphi\left(\mathbf{x}_{i}\right) .
$$

Based on the circulant matrix structure and Convolution Theorem, vector $\boldsymbol{\alpha}$ can be obtained by

$$
\boldsymbol{\alpha}=\mathcal{F}^{-1}\left(\frac{\mathcal{F}(\mathbf{y})}{\mathcal{F}(\mathbf{k})+\lambda}\right),
$$

where $\mathcal{F}$ and $\mathcal{F}^{-1}$ denote the Fourier transform and its inverse, respectively; $\mathbf{k}$ is a row vector of the $n \times n$ kernel matrix $\mathbf{K}$. Following the settings in KCF [5], the Gaussian kernel is adopted $\kappa\left(\mathbf{x}, \mathbf{x}^{\prime}\right)=\exp \left(-\left\|\mathbf{x}-\mathbf{x}^{\prime}\right\|^{2} / \sigma^{2}\right)$. And $\mathbf{k}$ is computed by

$$
\mathbf{k}=\exp \left(-\frac{1}{\sigma^{2}}\left(\|\mathbf{x}\|^{2}+\left\|\mathbf{x}^{\prime}\right\|^{2}-2 \mathcal{F}^{-1}\left(\mathcal{F}^{*}(\mathbf{x}) \cdot \mathcal{F}\left(\mathbf{x}^{\prime}\right)\right)\right)\right)
$$

where $\mathcal{F}^{*}(\mathbf{x})$ is the conjugate of the Fourier transform. The complexity of computing a full kernel correlation is $\mathcal{O}(n \log n)$ [5].

When tracking the object in subsequent frames, suppose the HOG feature of the candidate window is $\mathbf{z}$, the confidence score is calculated as

$$
y_{\mathbf{z}}=\sum_{i} \alpha_{i} \kappa\left(\mathbf{z}, \mathbf{x}_{i}\right)
$$

where $\kappa\left(\mathbf{z}, \mathbf{x}_{i}\right)$ is the kernel distance between regression sample $\mathbf{z}$ and training sample $\mathbf{x}_{i}$. Suppose $\mathbf{K}^{\mathbf{z}}$ is the kernel matrix composed of the kernel distance between all training samples and all candidate windows, it has been proved that $\mathbf{K}^{\mathbf{Z}}$ is a circulant matrix and the circulant basis is $\mathbf{k}^{\mathbf{x z}}$.

Similar to training, for the densely sampled candidate windows based on $\mathbf{z}$, we can obtain the following efficient computation based on convolution theorem

$$
\mathcal{F}(\mathbf{f}(\mathbf{z}))=\mathcal{F}\left(\mathbf{k}^{\mathbf{x z}}\right) \mathcal{F}(\boldsymbol{\alpha}),
$$




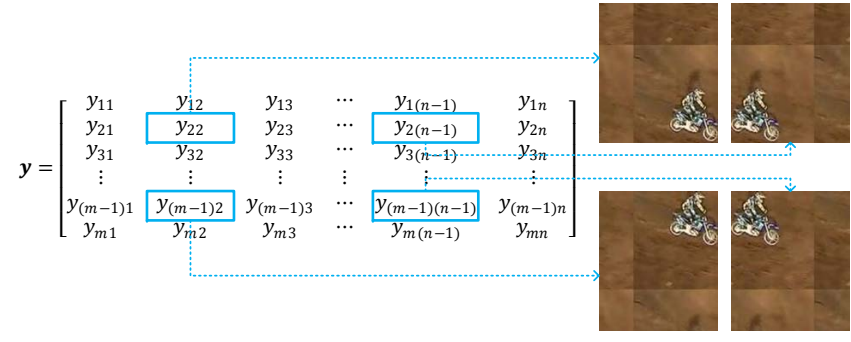

Fig. 3. The Gaussian training label matrix used in QKCF.

where $\mathbf{f}(\mathbf{z})$ contains the detection results of all the offset transform to candidate window $(\mathbf{z})$. The responses of all candidate offsets can be obtained by only one computation.

\section{B. Quadrangle Gaussian training label matrix}

In a traditional $\mathrm{KCF}$, the Gaussian training label matrix $\mathbf{y}$ used to train the filtering template is composed of the training labels with different offsets of the training samples. The $(i, j)$ element $y_{i j}$ is the label score of the offset window with $i-1$ steps (pixels if grey scale feature is used and cells if HOG is used) downwards and $j-1$ steps rightwards offset, while $y_{11}$ corresponds to the original location of the target without offset. Gaussian function is adopted to represent $\mathbf{y}$ in KCF [5]. When training the tracking model in the first frame, only the zero-offset window is used as a positive sample.

However, since KCF tracker uses a target-centered window as the training sample, it can only detect the offset of the target between the current frame and previous one. It is unable to detect the scale variation when target expands or shrinks visually because its tracking model is not trained that way. To keep it simple, $\mathrm{KCF}$ [5] does not determine the scale variation besides the location. PBT tracker [16], LCT tracker [17] and DSST tracker [18] are CF-based trackers to solve the scale variation problem, but all of them choose to sacrifice speed advantage by sampling, training and filtering the candidate windows multiple times.

To solve the scale variation problem under KCF tracker's one-sampe-one-filter framework, we redefine the Gaussian training label matrix $\mathbf{y}$ by incorporating the edge information of the target into the tracking model. Instead of only using the zero-offset (target-in-center) window as the positive sample, the offset images in the four window corners of the target are also considered as positive samples to determine the target edges. As shown in Figure 3 , the four elements $y_{22}, y_{2(n-1)}$, $y_{(m-1) 2}$ and $y_{(m-1)(n-1)}$ indicate the training response of the target-at-right-down-corner (right-down for short) window, left-down window, right-up window and the left-up window, respectively. By redefining the training matrix $\mathbf{y}$, we do not need to extract the four windows, because their corresponding elements in $\mathbf{y}$ will help do the work when filtering.

Formally, suppose the circulant offset between the four cornered training samples and the original sample are $\Delta_{1}=$ $\left(i_{1}, j_{1}\right), \Delta_{2}=\left(i_{2}, j_{2}\right), \Delta_{3}=\left(i_{3}, j_{3}\right), \Delta_{4}=\left(i_{4}, j_{4}\right)$, then the

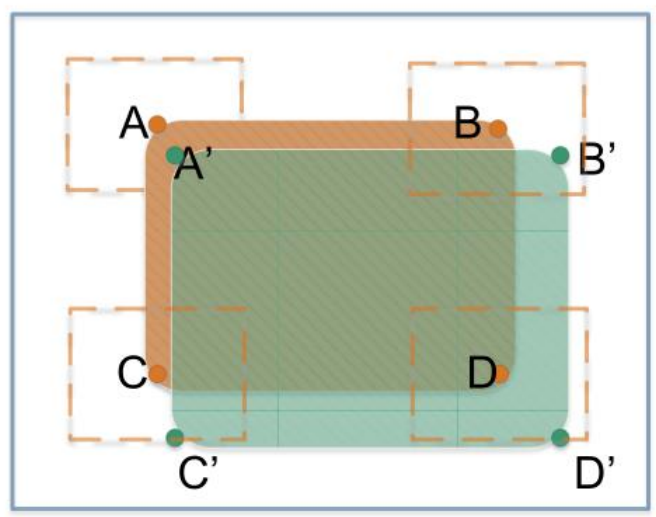

Fig. 4. Examples of quadrangle edge tracking.Rectangle $\mathrm{ABCD}$ is location of the current target, while $A^{\prime} B^{\prime} C^{\prime} D^{\prime}$ refers to target window of the next frame. The hollow blue rectangle represents the actual extended filter template. Nonzero areas of label matrix are shown as four dashed boxes.

matrix $\mathbf{y}$ can be calculated by

$$
\mathbf{y}=\sum_{i=1}^{4} G L(\widehat{\mathbf{y}}) \oplus \Delta_{i},
$$

where $G L(\widehat{\mathbf{y}})$ transforms the zero matrix $\widehat{\mathbf{y}}$ to a single peak Gaussian matrix where the single peak appears on the upper left $\widehat{y}_{11}, \oplus$ represents the circulant offset operation to a matrix. Inputting the redefined Gaussian training label matrix $\mathbf{y}$ into the tracking model allows us to train the KCF with the aim to obtain the four edged offset images. The size and location of the target can thereby be determined by utilizing the relative offset of the adjacent edged images.

As shown in Figure 4 rectangle $\mathrm{ABCD}$ represents the location of target in the current frame, while $\mathrm{A}^{\prime} \mathrm{B}^{\prime} \mathrm{C}^{\prime} \mathrm{D}$ ' refers to the its location in the next frame. It can be seen from the picture that the target moves towards right bottom direction and becomes a little larger.

The hollow blue rectangle represents the actual extended filter template. In the training stage, we use the patch cropped by blue rectangle to train the filter. The positive samples whose value are 1 locate at the very center of four corners of ABCD. To be more specifically, the whole label matrix $\mathbf{y}$ is defined by four accumulative Gaussian matrices. Here, nonzero areas are shown as four dashed boxes.

In detection, the detecting window is still the blue rectangle. We first get all the candidate windows by circulant dense matrices, which will be scanned via the filter we obtained in the training procedure. The one with the highest score is chosen as the final target of the current frame. According to the responsive matrix, we can now calculate the exact coordinates of the four border lines of the target rectangle. The detailed inference is provided in the next section.

\section{WEIGHTED BAYESIAN INFERENCE}

After the filtering operation of the scalable quadrangle single filter tracking, we can obtain a quadrangle response matrix. Apparently, the single peak localization method used in the traditional KCF [5] cannot work in our situation anymore. 
A scheme is needed to measure the confidence score of each partial tracking result based on both temporal and spatial information, and on top of it, a combining mechanism that determines the optimal target window by analysing multiple peak filtering results, each being elaborated below.

\section{A. Spatio-Temporal Confidence Score of Partial Filter Re- sponse}

Using the quadrangle Gaussian training label matrix $\mathbf{y}$ and the trained filter template $\mathcal{F}(\boldsymbol{\alpha})$, we can obtain the quadrangle filter response matrix $\mathbf{f}(\mathbf{z})$. In order to evaluate the filter response of each part, based on the circulant offset of $\mathbf{f}(\mathbf{z})$ with distance $\Delta_{i}=\left(i_{i}, j_{i}\right)$, we capture local response matrix $\mathbf{r}_{i}$ with width and height $s$ from the migrated matrix by

$$
\mathbf{r}_{i}=\left(\mathbf{f}(\mathbf{z}) \oplus \Delta_{i}\right)\left[-\frac{s}{2}: \frac{s}{2}\right] .
$$

Temporally, the movement of the target between two adjacent frames should not be large. As a result, we simply exploit filter response displacement to measure the smoothness of the tracking results between two frames for each part

$$
S C_{i}=\left\|\mathbf{r}_{i}^{t}-\mathbf{r}_{i}^{t-1}\right\|
$$

where $\mathbf{r}_{i}^{t}$ and $\mathbf{r}_{i}^{t-1}$ are the filter response matrix of the $i$ th part in the $t$ th and $t-1$ th frame.

Spatially, we use peak-to-sidelobe ratio (PSR) to measure the confidence score of the filter response peak to other candidate windows in the current frame, which is defined as

$$
P S R_{i}=\frac{\max \left(\mathbf{r}_{i}\right)-\mu_{i}}{\sigma_{i}},
$$

where $\mu_{i}$ and $\sigma_{i}$ are the average and standard deviation of $\mathbf{r}_{i}$, respectively. By jointly combining $S C_{i}$ and $P S R_{i}$, the likelihood of a partial detection being a true detection can be formulized as a weight, which can be calculated:

$$
\omega_{i}=\frac{1}{S C_{i}}+P S R_{i}
$$

\section{B. Maximum Posterior Probability of Target Window}

Let $s=(t, b, l, r)$ denote the bounding box of the target in the current frame, $s^{1}=(t, l), s^{2}=(t, r), s^{3}=(b, 1)$ and $s^{4}=(b, r)$ are the upper left, upper right, lower left and lower right coordinates of the window. Suppose $O=\left(o^{1}, o^{2}, o^{3}, o^{4}\right)$ is the filter result of the candidate windows in the current frame by QKCF, where $o^{i}=\left(\mathbf{r}_{i}, w_{i}\right)$. The optimal $s$ can be obtained by

$$
\begin{gathered}
s=\arg \min _{s_{j}} p\left(s_{j} \mid O\right), \\
p\left(s_{j} \mid O\right)=p\left(t_{j}, b_{j}, l_{j}, r_{j} \mid o^{1}, o^{2}, o^{3}, o^{4}\right) .
\end{gathered}
$$

Obviously, $t, b, l$ and $r$ are determined by $o^{1}$ and $o^{2}, o^{3}$ and $o^{4}, o^{1}$ and $o^{3}$, and $o^{2}$ and $o^{4}$, respectively. Take $t$ for an example, using Eq. (12), we can obtain

$$
t^{1}=\arg \min _{t_{j}^{1}} p\left(t_{j}^{1} \mid o^{1}\right),
$$

where $p\left(t_{j}^{1} \mid o^{1}\right)$ can be calculated by Bayesian

$$
p\left(t_{j}^{1} \mid o^{1}\right)=\frac{p\left(o^{1} \mid t_{j}^{1}\right) p\left(t_{j}^{1}\right)}{p\left(o^{1}\right)} \propto p\left(o^{1} \mid t_{j}^{1}\right) p\left(t_{j}^{1}\right) .
$$

As we use the $\mathrm{KCF}$ model, a filter response matrix is generated in each frame. The elements in the matrix represent the response score for some offset, which can be used to approximate $p\left(o^{1} \mid t_{j}^{1}\right) p\left(t_{j}^{1}\right)$

$$
\begin{aligned}
p\left(o^{1} \mid t_{j}^{1}\right) p\left(t_{j}^{1}\right) & \propto p\left(\mathbf{r}_{1}, w_{1} \mid t_{j}^{1}\right)=w_{1} p\left(\mathbf{r}_{1} \mid t_{j}^{1}\right) \\
& =w_{1} \max \left(\mathbf{r}_{1}\left(t_{j}^{1},:\right)\right) .
\end{aligned}
$$

Based on the smoothness constraint of adjunct frames, the prior probability $p\left(t_{j}^{1}\right)$ is defined as

$$
p\left(t_{j}^{1}\right)=\frac{h-\left|t_{j}^{1}-a_{2}\right|}{h}
$$

where $h$ is the width of a candidate window, $a_{2}$ is the coordinate of the upper left point in the target bounding box used for training filter template.

Combining Eq. (15), Eq. (16) and Eq. (17) will bring us:

$$
p\left(t_{j}^{1} \mid o^{1}\right)=\omega_{1} \max \left(\mathbf{r}_{1}\left(t_{j}^{1},:\right)\right) \frac{h-\left|t_{j}^{1}-a_{2}\right|}{h} .
$$

Similarly, we can obtain $p\left(t_{j}^{2} \mid o^{2}\right)$. Combining $p\left(t_{j}^{1} \mid o^{1}\right)$ and $p\left(t_{j}^{2} \mid o^{2}\right)$ with weight, we can finally obtain $t$ by

$$
t=\frac{t^{1} p\left(t_{j}^{1} \mid o^{1}\right)+t^{2} p\left(t_{j}^{2} \mid o^{2}\right)}{p\left(t_{j}^{1} \mid o^{1}\right)+p\left(t_{j}^{2} \mid o^{2}\right)} .
$$

The solution of $b, l, r$ can be similarly solved.

\section{Weighted Interpolation Updating}

To tackle the challenge of stability-plasticity dilemma, similar to KCF [5], we adopt a weighted interpolation updating scheme. The idea is that after obtaining the new location and size of the target, the predicted window is used as a positive sample to retrain the tracking model. The trained parameter $\mathcal{F}(\widehat{\boldsymbol{\alpha}})$ and feature $\widehat{\mathbf{x}}$ are incorporated into the original model parameter $\mathcal{F}(\boldsymbol{\alpha})$ and feature $\mathbf{x}$ by linear weights. Suppose the average and standard deviation of weights in Eq. (11) are $\bar{\omega}$ and $\omega_{\sigma}$, which can reflect the tracking results of $\mathcal{F}(\boldsymbol{\alpha})$. We propose to compute the overall confidence estimation $\omega$ of the model in the current frame by

$$
\omega=\bar{\omega}+\frac{\beta}{\omega_{\sigma}},
$$

where $\beta$ is used to control the ratio between $\bar{w}$ and $w_{\sigma}$. The weighted interpolation updating model is

$$
\begin{gathered}
\mathcal{F}(\boldsymbol{\alpha})=(1-\mu \omega) \mathcal{F}(\boldsymbol{\alpha})+\mu \omega \mathcal{F}(\widehat{\boldsymbol{\alpha}}), \\
\mathbf{x}=(1-\mu \omega) \mathbf{x}+\mu \omega \widehat{\mathbf{x}},
\end{gathered}
$$

where $\mu$ is the parameter to control the updating ratio. With the weighted interpolation updating scheme, QKCF can well handle the problems, such as the light and shadow changes and partial occlusion. When processing sequences with poor tracking results, weight $\omega$ tends to be 0 , which can avoid incorporating wrong data into the tracking model. The details are given in Algorithm 1

\section{COMPLEXITY ANALYSIS}

QKCF provides a method that can obtain the four corners of target in one single filtering operation instead of adding 


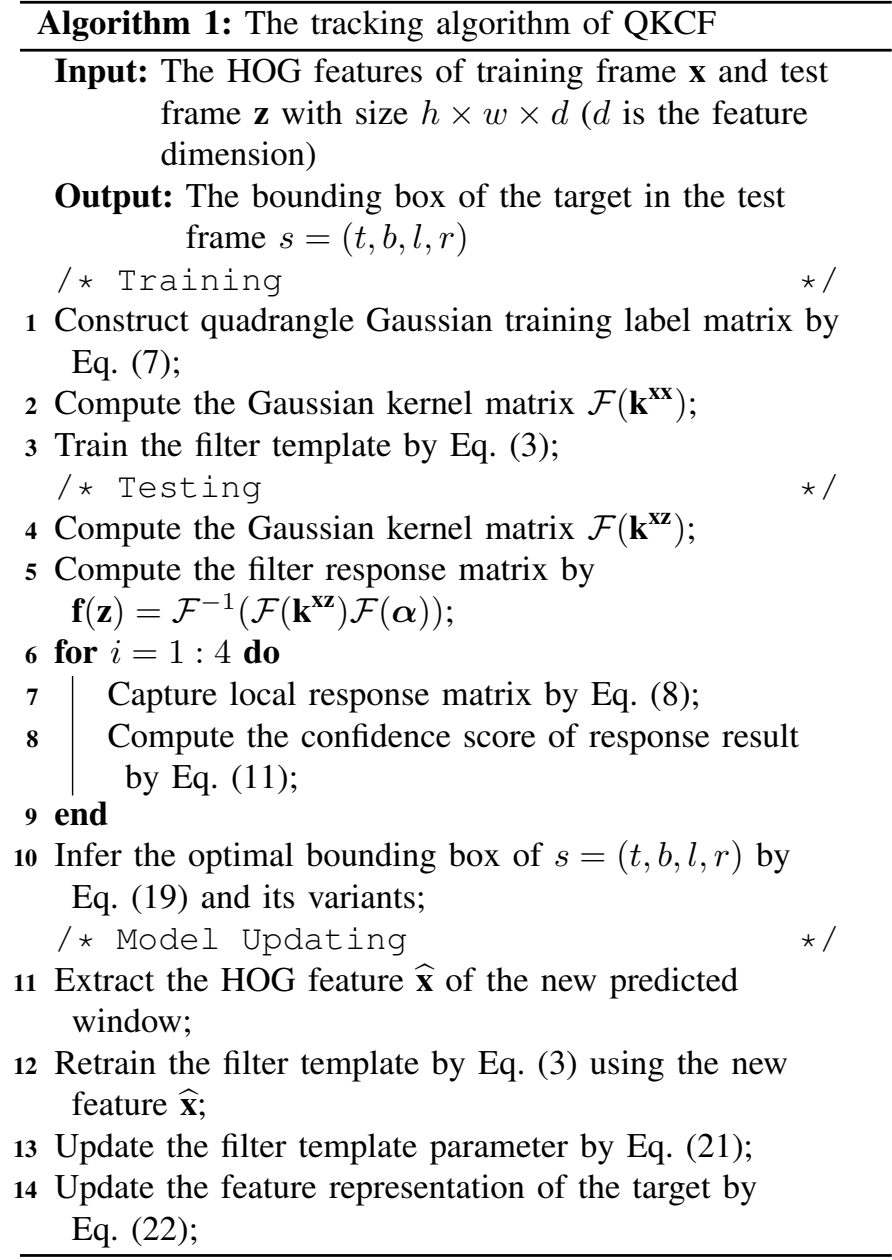

extra filters like part-based tracker. As in Algorithm 1, the whole process can be divided into 4 parts, including training, testing, inference and model updating. Approximately, in the phase of training and testing, the complexity of training should be the same as the original KCF [5]. In the inference phase, when calculating the exact coordinates of the target based on weighted Bayesian inference, our method brings in an extra cost of $\mathcal{O}\left(s^{2}\right)$, where $s$ is the side length of the local response matrix. Compared to the overall costs of training and testing, this extra cost is rather small and can be negligible. In the model updating phase, though our strategy is slightly different from KCF [5], calculating an extra weight parameter $\mathbf{w}$ as Eq. 20) specified has a cost of $\mathrm{O}(1)$. Therefore, QKCF is equivalent to $\mathrm{KCF}[5]$ in terms of the complexity. This is confirmed by the results reported in Table. IV, where the obtained FPSs (frames per second) of two algorithms are 168 and 195 , respectively.

\section{EXPERIMENTS}

To validate the effectiveness of the proposed QKCF method for visual tracking, we carry out extensive experiments in both scalable and unscalable situations with comparisons to stateof-the-art methods.
TABLE I

THE AVERAGE TRACKING PERFORMANCE COMPARISON OF THE PROPOSED METHOD WITH STATE-OF-THE-ART METHODS ON THE OTB-100 DATASET MEASURED BY CLE AND F/S.

\begin{tabular}{|r|c|c|c|c|}
\hline OTB-100 & QKCF & KCF [5] & DSST [18] & LCT [17] \\
\hline \hline CLE & 40.6 & 40.4 & 40.9 & 38.2 \\
f/s & 165.5 & 198 & 29.9 & 17.6 \\
\hline
\end{tabular}
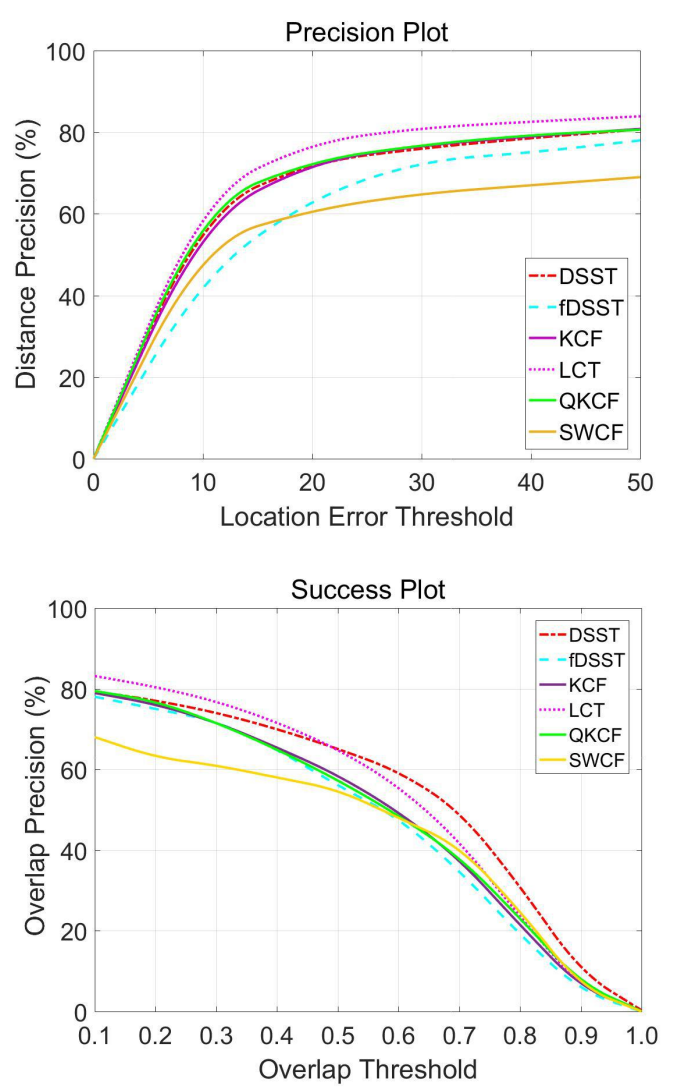

Fig. 5. Precision and success plots over the 100 video sequences in the OTB-100 dataset.

\section{A. Datasets}

For evaluation, we employ 100 video sequences in OTB100, a large object tracking benchmark dataset [6], covering all difficult situations, such as illumination variation, scale variation, occlusion, background clutter and motion blur. To better compare the performance on scalable tracking, we select 16 sequences from OTB-100, where severe scale variations arise.

\section{B. Experimental Settings}

The proposed QKCF algorithm is implemented in Matlab and evaluated on a desktop with an Intel (R) Core (TM) i5$34703.20 \mathrm{GHz}$ CPU and 8 GB RAM.

Evaluation Metric. The tracking performance is typically evaluated on the localization precision and bounding box overlap rate. We use centre location error (CLE) and precision plot to measure the localization precision. CLE is computed as the average Euclidean distance between the ground-truth and the 


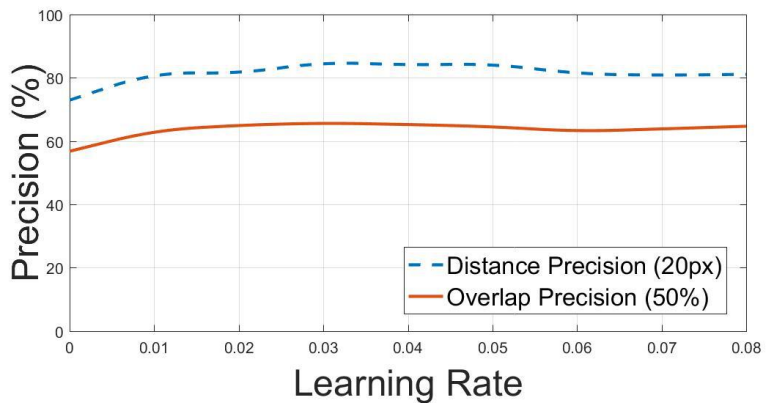

Fig. 6. Performance of different learning rate $\mu$ on OTB-100.

estimated centre location of the target object, while the average distance precision is plotted over a range of thresholds in the precision plot. Following the PASCAL evaluation criteria [43], success plot is used to measure bounding box overlap rate. Finally, we provide the visualized qualitative analysis of our approach with comparison to existing tracking methods.

Baseline Methods. On the overall tracking in OTB-100, we tested 5 state-of-the-art trackers. They are KCF [5], SWCF [45], DSST [18], fDSST [44] and LCT [17], where the last three ones are scalable trackers. On the scalable dataset, we use 4 trackers of them as baseline which includes KCF [5], DSST [18], fDSST [44], LCT [17].

Implementation Details. Empirically, $\beta$ in Eq. 20 is set to 1 , while the learning rate $\mu$ in Eq. 21) is set to 0.03 . As in [18], we use densely sampled HOG [46] for image representation. Using a cell size of $4 \times 4$, we can extract the features with length 992, which are always multiplied by a Hann window [7].

\section{On the Overall Tracking in OTB-100}

The average performance on CLE and the computational efficiency for the 100 video sequences tested on the OTB-100 dataset is shown in Table [1 and its corresponding precision and success plot are shown in Figure 5. From Table [1] we can find that the six trackers perform competitively for the unscalable tracking. It reveals that $\mathrm{KCF}$ [5] and its variants perform stably when tracking objects in most situations.

Seen from Figure 5. we can observe that the performance of QKCF is similar to that of KCF [5], and is higher than fDSST [44] and SWCF [45], while DSST [18] and LCT [17] perform better than the proposed QKCF and unscalable KCF [5] on overlap precision in the OTB-100 dataset. However, the better performance of DSST [18] and LCT [17] comes with the great sacrifice of computational efficiency, which is confirmed by the fact that QKCF tracker is 5 to 10 times faster than DSST [18] and LCT [17]. The reason behind is that only one filtering operation is required for each frame in QKCF, while one 3D template based filtering and 33 2D template based filtering are operated on the default 33-layer spatial pyramid for DSST [18] and LCT [17], respectively.

Additionally, we also conduct sensitivity tests when varying parameter $\beta$ in Eq. 20, and the learing rate $\mu$ in Eq. 21. Because the standard deviation of weights is so small in most
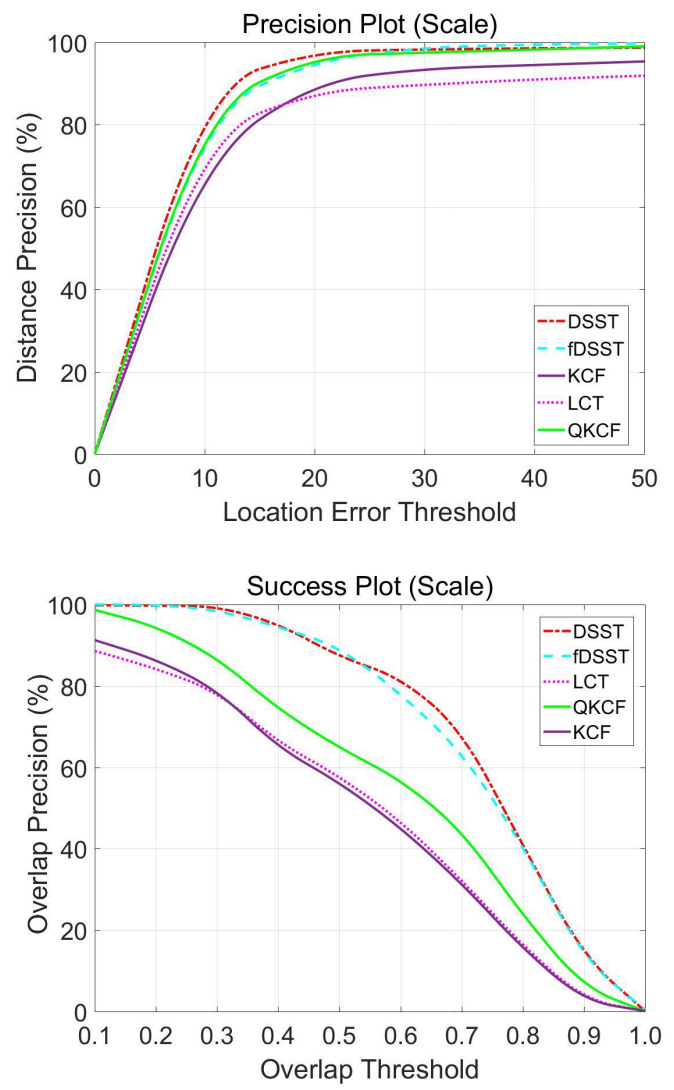

Fig. 7. Precision and success plots over the 16 video sequences with significant scale variations.

cases that the results are not that sensitive to $\beta$, which can be simply set to 1 . As for the learning rate $\mu$, the results are shown in Figure 6

\section{On Scalable Visual Tracking}

The average performance of CLE and the computational efficiency for the 16 video sequences with significant scale variations is shown in Table IV, while the performance for each sequence is shown in Table [I] and Table [III respectively. The precision and success plot are shown in Figure 7

We can find from Table II that the proposed QKCF method achieves the best performance in 3 of the 16 sequences on CLE, while DSST [18] , LCT [17] and fDSST [44] perform best on 5, 3, 4 sequences, respectively. Further, the CLE of QKCF is relatively stable without significant changes. KCF [5] is the most sensitive to scale variation, which indicates that KCF cannot well handle the scale variation. The results demonstrate the effectiveness and robustness of QKCF for scalable tracking.

From Table IV, it is clear that the tracking speed of QKCF is 5 to 8 times faster than DSST [18] and LCT [17] for scalable tracking. From Table III, we can find that the proposed QKCF method achieves the best performance in 4 of the 16 sequences on computational efficiency, while KCF [5] performs best on the other 12 sequences. Even on the challenging Car4 sequence with high resolution, QKCF can process $63.8 \mathrm{f} / \mathrm{s}$, exceeding the real-time requirement, while DSST [18] and 
TABLE II

THE PERFORMANCE COMPARISON OF THE PROPOSED METHOD WITH STATE-OF-THE-ART METHODS ON SCALABLE VISUAL TRACKING MEASURED BY CLE. THE BEST PERFORMANCES ARE HIGHLIGHTED BY BOLD.

\begin{tabular}{|r|c|c|c|c|c|c|c|c|c|c|c|c|c|c|c|}
\hline CLE & $\begin{array}{c}\text { Blur } \\
\text { Body }\end{array}$ & Car1 & Car2 & Car24 & Car4 & $\begin{array}{c}\text { Car } \\
\text { Scale }\end{array}$ & $\begin{array}{c}\text { Cros } \\
\text { sing }\end{array}$ & Dog1 & $\begin{array}{c}\text { Sing } \\
\text { er1 }\end{array}$ & $\begin{array}{c}\text { Sing } \\
\text { er2 }\end{array}$ & $\begin{array}{c}\text { Skat } \\
\text { ing1 }\end{array}$ & $\begin{array}{c}\text { Sylve } \\
\text { ster }\end{array}$ & $\begin{array}{c}\text { Twin } \\
\text { Toy }\end{array}$ & $\begin{array}{c}\text { Walk } \\
\text { ings }\end{array}$ & $\begin{array}{c}\text { Walk } \\
\text { ing1 }\end{array}$ \\
ing2
\end{tabular}

TABLE III

THE EFFICIENCY COMPARISON OF THE PROPOSED METHOD WITH STATE-OF-THE-ART METHODS ON SCALABLE VISUAL TRACKING MEASURED BY F/S. THE HIGHEST EFFICIENCY IS HIGHLIGHTED BY BOLD.

\begin{tabular}{|c|c|c|c|c|c|c|c|c|c|c|c|c|c|c|c|c|}
\hline f/s & $\begin{array}{l}\text { Blur } \\
\text { Body }\end{array}$ & Car 1 & Car2 & Car24 & Car4 & $\begin{array}{c}\text { Car } \\
\text { Scale }\end{array}$ & $\begin{array}{l}\text { Cros } \\
\text { sing }\end{array}$ & Dog1 & $\begin{array}{c}\text { Sing } \\
\text { er1 }\end{array}$ & $\begin{array}{c}\text { Sing } \\
\text { er2 }\end{array}$ & $\begin{array}{l}\text { Skat } \\
\text { ing1 }\end{array}$ & $\begin{array}{l}\text { Sylve } \\
\text { ster }\end{array}$ & Toy & $\begin{array}{l}\text { Twin } \\
\text { ings }\end{array}$ & $\begin{array}{l}\text { Walk } \\
\text { ing } 1\end{array}$ & $\begin{array}{l}\text { Walk } \\
\text { ing2 }\end{array}$ \\
\hline QKCF & 87.1 & 141 & 155 & 290 & 63.8 & 264 & 303 & 188 & 118 & 148 & 152 & 157 & 123 & 133 & 217 & 147 \\
\hline $\mathrm{KCF} \mid 5$ & 108 & 131 & 198 & 356 & 74.7 & 331 & 356 & 203 & 108 & 200 & 158 & 156 & 131 & 233 & 251 & 133 \\
\hline DSST [18] & 3.75 & 27.8 & 29.9 & 41.4 & 10.2 & 46.6 & 64.3 & 63.2 & 4.22 & 29.8 & 25.8 & 30.6 & 20.9 & 53.6 & 42.8 & 25.0 \\
\hline LCT & 12.3 & 14.8 & 9.12 & 29.0 & 11.5 & 22.7 & 35.6 & 9.54 & 17.9 & 28.2 & 10.8 & 17.1 & 13.2 & 28.0 & 30.1 & 21.6 \\
\hline fDSST & 15.3 & 102 & 113 & 145 & 64.6 & 85.7 & 129 & 124 & 21 & 10.5 & 97.7 & 112 & 118 & 75.1 & 111 & 86.5 \\
\hline
\end{tabular}
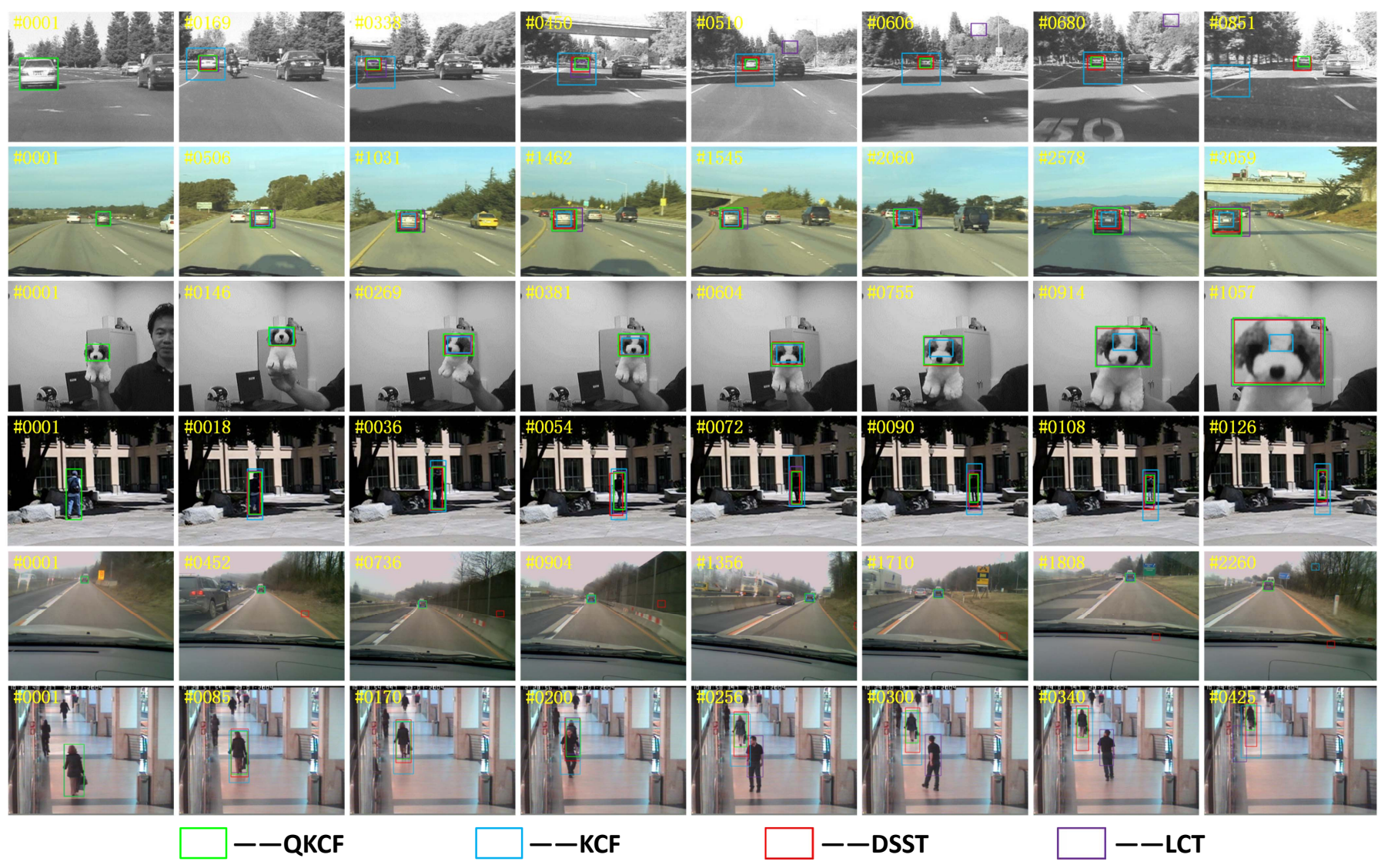

Fig. 8. Tracking results of our QKCF algorithm, KCF [5], DSST [18] and LCT [17] methods on six challenging sequences (from top to bottom are Carl, Car24, Dog1, Human8, Volkswagen and Walking2, respectively).

LCT [17] can track the object with $30 \mathrm{f} / \mathrm{s}$, which may not be used for real-time tracking. On the sequences with obvious shaking (beginning with Blur) and light and shadow changes (Carl), the tracking speeds of both DSST [18] and LCT [17] are below $20 \mathrm{f} / \mathrm{s}$, while QKCF can process $120 \mathrm{f} / \mathrm{s}$, which is robust to shaking and light and shadow changes. Compared with $\mathrm{KCF}$ [5], the efficiency of QKCF is slightly lower, which is caused by the weighted Bayesian inference in Section IV
The precision plot shows that the proposed QKCF outperforms KCF [5] and LCT [17], demonstrating great stability and robustness of QKCF for scalable tracking. Viewing the results when the location error threshold is set to 20 as previous methods, we can find that the distance precision achieves 96.7\%, while the precisions of KCF [5], DSST [18], LCT [17] and fDSST [44] are 90.4\%,97.8\%,88.1\% and $96.7 \%$, respectively. The precision improvement of QKCF over 
TABLE IV

THE AVERAGE EFFICIENCY COMPARISON OF THE PROPOSED METHOD WITH STATE-OF-THE-ART METHODS ON THE 16 SCALABLE SEQUENCES MEASURED BY F/S.

\begin{tabular}{|r|c|c|c|c|c|}
\hline Scalable & QKCF & KCF & $\begin{array}{r}{[\overline{18}]} \\
\text { DSST }\end{array}$ & $\begin{array}{c}{[17]} \\
\text { LCT }\end{array}$ & $\begin{array}{c}{[44]} \\
\text { fDSST }\end{array}$ \\
\hline \hline CLE & 6.2 & 11.5 & 5.4 & 17.7 & 5.6 \\
f/s & 168 & 195 & 32.4 & 19.5 & 88 \\
\hline
\end{tabular}

$\mathrm{KCF}$ is $6.92 \%$. Therefore, we can conclude that the proposed QKCF significantly outperforms KCF on distance precision.

It is clear from the success plot that the proposed QKCF outperforms KCF [5] and LCT [17], but performs very slightly worse than DSST [18] and fDSST [44]. When setting the overlap threshold to 0.5 , the overlap precision of QKCF is $64.8 \%$, while the precisions of unscalable KCF [5], DSST [18], LCT [17] and fDSST [44] are 56.3\%, 87.2\%, 57.8\% and $87.1 \%$, respectively. The performance improvement of QKCF over $\mathrm{KCF}$ is $15.1 \%$, from which we can again clearly view the superiority of QKCF over KCF.

To better show the tracking results with scale variations, we compare our algorithm with the other three state-of-the-art trackers (KCF [5], scalable DSST [18] and LCT [17]) on 6 challenging sequences, as shown in Figure 8

From the above analysis, we can conclude that by sacrificing little computational efficiency, the proposed QKCF can significantly improve the distance precision and overlap precision for scalable tracking in a single frame single filter framework. The superior tracking precision and high computational efficiency make QKCF more practical than DSST [18] and LCT [17] for real-time tracking with significant scale variations.

\section{E. Performance comparison of peak numbers}

In principle, 2-peaks filter can already detect the location as well as the scale of object simultaneously. In this part of experiment, we intend to validate the effectiveness of 4-peaks strategy, compared to other numbers of peak. Therefore, apart from our QKCF (4-peaks filter) and the KCF (1-peak filter), we also include in the experiment two 2-peaks filters, one 3peaks filter and one 5-peaks filter. Specifically, the first 2-peaks filter has positive samples in left-top and right-bottom corners (LT-RB for short) while the second 2-peaks filter has positive samples in LB-RT corners. Similarly, the 3-peaks filter has positive samples at LT-LB-RB corners, and the 5-peaks filter is a quadrangle filter plus a single peak in the center.

As for the estimation of the target window, we use a similar weighted Bayesian inference strategy. Specially, for 5-peaks filter we first use the same method to calculate the target window as quadrangle filter does, then adjust the location of the whole target window according to the additional center peak. Figure 9 depicts the obtained result.

Seen from the result, it is clear that the 4-peaks filter outperforms all the other peaks strategies. To be precise, two 2-peaks filters are far behind the 4-peaks filter; 5-peaks filter shows better precision than 2-peaks and 3-peaks strategies but is still slightly lower than 4-peaks filter in terms of the
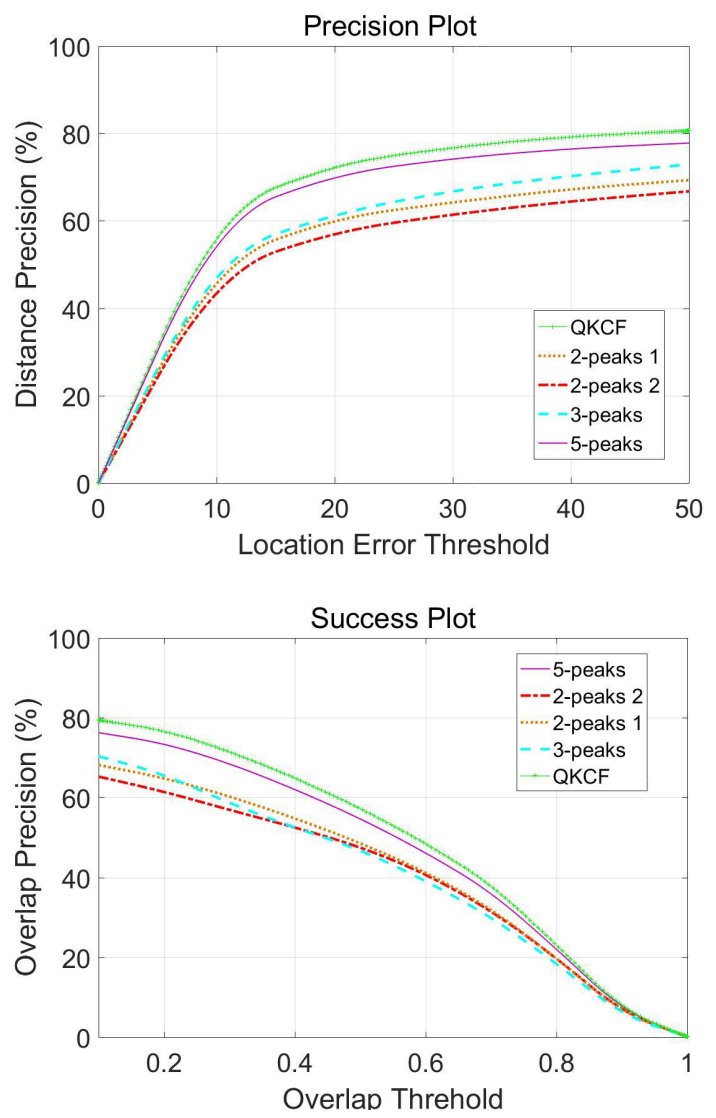

Fig. 9. Precision and success plots over different numbers of peak.

performance. In general, more peaks lead to higher accuracy and better stability, because more information from various peaks is involved in estimating the bounding box of object. That interprets why 4-peaks filter looks much better than 2peaks and 3-peaks filters. However, when we add an additional peak to the center, the precision gets lower on the contrary, which means the fifth peak is redundant and may provide useless information. The result turns out that choice of 4 peaks is a good trade-off between algorithm efficiency and accuracy.

\section{CONCLUSION AND FutURE WORK}

In this paper, we studied how to utilize the Gaussian training label matrix in KCF to solve the scale variation problem in single filter tracking. By utilizing the circulant matrix structure of the densely sampled candidate bounding box in $\mathrm{KCF}$, we proposed a quadrangle Gaussian training label matrix to incorporate the location and size estimation problem into one filtering operation. By sacrificing little computational efficiency, the proposed QKCF can handle the scale variation in single filter tracking. A weighted Bayesian inference framework is then utilized to deduce the location and size of the bounding box. The experimental results demonstrate the effectiveness, efficiency and robustness of the proposed QKCF for both scalable and unscalable tracking. For further studies, we will try to replace the HOG feature with CNN representation [47]-[54] to fully explore the visual features. How to extend the proposed quadrangle Gaussian training 
label matrix to other applications, such as image retrieval [55][57], human action recognition [58], [59], and visual saliency detection [60], [61], is also worth studying.

\section{REFERENCES}

[1] A. Yilmaz, O. Javed, and M. Shah, "Object tracking: A survey," ACM Computing Surveys, vol. 38, no. 4, p. 13, 2006.

[2] A. W. M. Smeulders, D. M. Chu, R. Cucchiara, S. Calderara, A. Dehghan, and M. Shah, "Visual tracking: An experimental survey," IEEE Transactions on Pattern Analysis and Machine Intelligence, vol. 36, no. 7, pp. 1442-1468, 2014.

[3] H. Zhou, H. Kong, L. Wei, D. Creighton, and S. Nahavandi, "Efficient road detection and tracking for unmanned aerial vehicle," IEEE Transactions on Intelligent Transportation Systems, vol. 16, no. 1, pp. 297-309, 2015.

[4] J.-P. Jodoin, G.-A. Bilodeau, and N. Saunier, "Tracking all road users at multimodal urban traffic intersections," IEEE Transactions on Intelligent Transportation Systems, vol. 17, no. 11, pp. 3241-3251, 2016.

[5] J. F. Henriques, R. Caseiro, P. Martins, and J. Batista, "High-speed tracking with kernelized correlation filters," IEEE Transactions on Pattern Analysis and Machine Intelligence, vol. 37, no. 3, pp. 583-596, 2015.

[6] Y. Wu, J. Lim, and M.-H. Yang, "Online object tracking: A benchmark," in IEEE Conference on Computer Vision and Pattern Recognition (CVPR), 2013, pp. 2411-2418.

[7] D. S. Bolme, J. R. Beveridge, B. A. Draper, and Y. M. Lui, "Visual object tracking using adaptive correlation filters," in IEEE Conference on Computer Vision and Pattern Recognition (CVPR), 2010, pp. 2544 2550.

[8] J. F. Henriques, R. Caseiro, P. Martins, and J. Batista, "Exploiting the circulant structure of tracking-by-detection with kernels," in European conference on computer vision, 2012, pp. 702-715.

[9] M. Danelljan, F. Khan, M. Felsberg, and J. Weijer, "Adaptive color attributes for real-time visual tracking," in IEEE Conference on Computer Vision and Pattern Recognition (CVPR), 2014, pp. 1090-1097.

[10] K. Zhang, L. Zhang, Q. Liu, D. Zhang, and M.-H. Yang, "Fast visual tracking via dense spatio-temporal context learning," in European Conference on Computer Vision (ECCV), 2014, pp. 127-141.

[11] S. Hare, A. Saffari, and P. H. Torr, "Struck: Structured output tracking with kernels," in IEEE International Conference on Computer Vision (ICCV), 2011, pp. 263-270.

[12] Z. Kalal, K. Mikolajczyk, and J. Matas, "Tracking-learning-detection," IEEE Transactions on Pattern Analysis and Machine Intelligence, vol. 34, no. 7, pp. 1409-1422, 2012.

[13] B. Babenko, M.-H. Yang, and S. Belongie, "Robust object tracking with online multiple instance learning," IEEE Transactions on Pattern Analysis and Machine Intelligence, vol. 33, no. 8, pp. 1619-1632, 2011.

[14] Y. Wu, B. Shen, and H. Ling, "Online robust image alignment via iterative convex optimization," in IEEE Conference on Computer Vision and Pattern Recognition (CVPR), 2012, pp. 1808-1814.

[15] N. Dalal and B. Triggs, "Histograms of oriented gradients for human detection," in IEEE Conference on Computer Vision and Pattern Recognition (CVPR), 2005, pp. 886-893.

[16] T. Liu, G. Wang, and Q. Yang, "Real-time part-based visual tracking via adaptive correlation filters," in IEEE Conference on Computer Vision and Pattern Recognition (CVPR), 2015, pp. 4902-4912.

[17] C. Ma, X. Yang, C. Zhang, and M.-H. Yang, "Long-term correlation tracking," in IEEE Conference on Computer Vision and Pattern Recognition (CVPR), 2015, pp. 5388-5396.

[18] M. Danelljan, G. Häger, F. Khan, and M. Felsberg, "Accurate scale estimation for robust visual tracking," in British Machine Vision Conference $(B M V C), 2014$.

[19] S. Zhang, H. Yao, X. Sun, and X. Lu, "Sparse coding based visual tracking: Review and experimental comparison," Pattern Recognition, vol. 46, no. 7, pp. 1772-1788, 2013.

[20] D. S. Bolme, B. A. Draper, and J. R. Beveridge, "Average of synthetic exact filters," in IEEE Conference on Computer Vision and Pattern Recognition (CVPR), 2009, pp. 2105-2112.

[21] T. Lindeberg, "Scale selection properties of generalized scale-space interest point detectors," Journal of Mathematical Imaging and vision, vol. 46, no. 2, pp. 177-210, 2013.

[22] Z. Kalal, J. Matas, and K. Mikolajczyk, "Pn learning: Bootstrapping binary classifiers by structural constraints," in IEEE Conference on Computer Vision and Pattern Recognition (CVPR), 2010, pp. 49-56.
[23] M. Danelljan, A. Robinson, F. S. Khan, and M. Felsberg, "Beyond correlation filters: Learning continuous convolution operators for visual tracking," in European Conference on Computer Vision, 2016, pp. 472 488.

[24] S. Hong, T. You, S. Kwak, and B. Han, "Online tracking by learning discriminative saliency map with convolutional neural network," in International Conference on Machine Learning, 2015, pp. 597-606.

[25] C. Ma, J.-B. Huang, X. Yang, and M.-H. Yang, "Hierarchical convolutional features for visual tracking," in Proceedings of the IEEE International Conference on Computer Vision, 2015, pp. 3074-3082.

[26] L. Wang, W. Ouyang, X. Wang, and H. Lu, "Visual tracking with fully convolutional networks," in Proceedings of the IEEE International Conference on Computer Vision, 2015, pp. 3119-3127.

[27] H. Nam and B. Han, "Learning multi-domain convolutional neural networks for visual tracking," in Proceedings of the IEEE Conference on Computer Vision and Pattern Recognition, 2016, pp. 4293-4302.

[28] R. Tao, E. Gavves, and A. W. Smeulders, "Siamese instance search for tracking," in Proceedings of the IEEE Conference on Computer Vision and Pattern Recognition, 2016, pp. 1420-1429.

[29] J. Sanchez-Riera, K. Srinivasan, K.-L. Hua, W.-H. Cheng, M. A. Hossain, and M. F. Alhamid, "Robust rgb-d hand tracking using deep learning priors," IEEE Transactions on Circuits and Systems for Video Technology, 2017.

[30] S. Avidan, "Ensemble tracking," IEEE transactions on Pattern Analysis and Machine Intelligence, vol. 29, no. 2, 2007.

[31] Q. Bai, Z. Wu, S. Sclaroff, M. Betke, and C. Monnier, "Randomized ensemble tracking," in Proceedings of the IEEE International Conference on Computer Vision, 2013, pp. 2040-2047.

[32] N. Wang and D.-Y. Yeung, "Ensemble-based tracking: Aggregating crowdsourced structured time series data." in International Conference on Machine Learning (ICML), 2014, pp. 1107-1115.

[33] R. O. Chavez-Garcia and O. Aycard, "Multiple sensor fusion and classification for moving object detection and tracking," IEEE Transactions on Intelligent Transportation Systems, vol. 17, no. 2, pp. 525-534, 2016.

[34] X. Sun, H. Yao, S. Zhang, and D. Li, "Non-rigid object contour tracking via a novel supervised level set model," IEEE Transactions on Image Processing, vol. 24, no. 11, pp. 3386-3399, 2015.

[35] M. Demi, "Contour tracking with a spatio-temporal intensity moment," IEEE transactions on Pattern Analysis and Machine Intelligence, vol. 38, no. 6, pp. 1141-1154, 2016.

[36] J. Fang, H. Xu, Q. Wang, and T. Wu, "Online hash tracking with spatiotemporal saliency auxiliary," Computer Vision and Image Understanding, vol. 160, pp. 57-72, 2017.

[37] Q. Wang, J. Fang, and Y. Yuan, "Multi-cue based tracking," Neurocomputing, vol. 131, pp. 227-236, 2014.

[38] S.-H. Bae and K.-J. Yoon, "Robust online multi-object tracking based on tracklet confidence and online discriminative appearance learning," in IEEE Conference on Computer Vision and Pattern Recognition (CVPR), 2014, pp. 1218-1225.

[39] A. Vatavu, R. Danescu, and S. Nedevschi, "Stereovision-based multiple object tracking in traffic scenarios using free-form obstacle delimiters and particle filters," IEEE Transactions on Intelligent Transportation Systems, vol. 16, no. 1, pp. 498-511, 2015.

[40] L. Fagot-Bouquet, R. Audigier, Y. Dhome, and F. Lerasle, "Improving multi-frame data association with sparse representations for robust nearonline multi-object tracking," in European Conference on Computer Vision (ECCV). Springer, 2016, pp. 774-790.

[41] J. Hong Yoon, C.-R. Lee, M.-H. Yang, and K.-J. Yoon, "Online multiobject tracking via structural constraint event aggregation," in IEEE Conference on Computer Vision and Pattern Recognition (CVPR), 2016, pp. $1392-1400$.

[42] A. K. KC, L. Jacques, and C. De Vleeschouwer, "Discriminative and efficient label propagation on complementary graphs for multiobject tracking," IEEE transactions on Pattern Analysis and Machine Intelligence, vol. 39, no. 1, pp. 61-74, 2017

[43] M. Everingham, L. Van Gool, C. K. Williams, J. Winn, and A. Zisserman, "The pascal visual object classes (voc) challenge," International Journal of Computer Vision, vol. 88, no. 2, pp. 303-338, 2010.

[44] M. Danelljan, G. Häger, F. S. Khan, and M. Felsberg, "Discriminative scale space tracking," IEEE transactions on pattern analysis and machine intelligence, vol. 39, no. 8, pp. 1561-1575, 2017.

[45] E. Gundogdu and A. A. Alatan, "Spatial windowing for correlation filter based visual tracking," in IEEE International Conference on Image Processing (ICIP), 2016, pp. 1684-1688.

[46] P. F. Felzenszwalb, R. B. Girshick, D. McAllester, and D. Ramanan, "Object detection with discriminatively trained part-based models," 
IEEE Transactions on Pattern Analysis and Machine Intelligence, vol. 32 , no. 9, pp. $1627-1645,2010$

[47] J. Fan, W. Xu, Y. Wu, and Y. Gong, "Human tracking using convolutional neural networks," IEEE Transactions on Neural Networks, vol. 21, no. 10, pp. 1610-1623, 2010.

[48] N. Wang and D.-Y. Yeung, "Learning a deep compact image representation for visual tracking," in Advances in Neural Information Processing Systems (NIPS), 2013, pp. 809-817.

[49] S. Hong, T. You, S. Kwak, and B. Han, "Online tracking by learning discriminative saliency map with convolutional neural network." in International Conference on Machine Learning (ICML), 2015, pp. 597606.

[50] Y. Qi, S. Zhang, L. Qin, H. Yao, Q. Huang, J. Lim, and M.-H. Yang, "Hedged deep tracking," in Proceedings of the IEEE Conference on Computer Vision and Pattern Recognition, 2016, pp. 4303-4311.

[51] J. Gao, Q. Wang, and Y. Yuan, "Embedding structured contour and location prior in siamesed fully convolutional networks for road detection," IEEE Transactions on Intelligent Transportation Systems, 2017.

[52] Q. Wang, J. Gao, and Y. Yuan, "A joint convolutional neural networks and context transfer for street scenes labeling," IEEE Transactions on Intelligent Transportation Systems, 2017.

[53] Q. Wang, J. Wan, and Y. Yuan, "Deep metric learning for crowdedness regression," IEEE Transactions on Circuits and Systems for Video Technology, 2017.

[54] S. Zhao, H. Yao, Y. Gao, R. Ji, and G. Ding, "Continuous probability distribution prediction of image emotions via multitask shared sparse regression," IEEE Transactions on Multimedia, vol. 19, no. 3, pp. 632645, 2017.

[55] Y. Guo, G. Ding, L. Liu, J. Han, and L. Shao, "Learning to hash with optimized anchor embedding for scalable retrieval," IEEE Transactions on Image Processing, vol. 26, no. 3, pp. 1344-1354, 2017.

[56] Z. Lin, G. Ding, J. Han, and J. Wang, "Cross-view retrieval via probability-based semantics-preserving hashing," IEEE Transactions on Cybernetics, vol. 47, no. 12, pp. 4342-4355, 2017.

[57] Y. Guo, G. Ding, J. Han, and Y. Gao, "Zero-shot learning with transferred samples," IEEE Transactions on Image Processing, vol. 26, no. 7, pp. 3277-3290, 2017.

[58] B. Zhang, A. Perina, Z. Li, V. Murino, J. Liu, and R. Ji, "Bounding multiple gaussians uncertainty with application to object tracking," International Journal of Computer Vision, vol. 118, no. 3, pp. 364-379, 2016.

[59] B. Zhang, Y. Yang, C. Chen, L. Yang, J. Han, and L. Shao, "Action recognition using $3 \mathrm{~d}$ histograms of texture and a multi-class boosting," IEEE Transactions on Image Processing, vol. 26, no. 10, pp. 4648-4660, 2017.

[60] D. Zhang, J. Han, L. Jiang, S. Ye, and X. Chang, "Revealing event saliency in unconstrained video collection," IEEE Transactions on Image Processing, vol. 26, no. 4, pp. 1746-1758, 2017.

[61] X. Yao, J. Han, D. Zhang, and F. Nie, "Revisiting co-saliency detection: a novel approach based on two-stage multi-view spectral rotation coclustering,", IEEE Transactions on Image Processing, vol. 26, no. 7, pp. 3196-3209, 2017

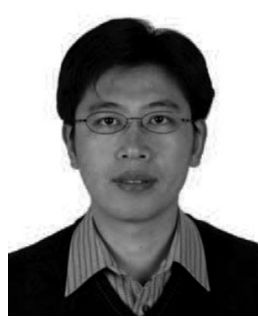

Guiguang Ding received the Ph.D. degree in electronic engineering from Xidian University, China. $\mathrm{He}$ is currently an Associate Professor with the School of Software, Tsinghua University. Since 2006, he has been a postdoctoral research fellow with the Department of Automation, Tsinghua University. His current research centers on the area of multimedia information retrieval, computer vision, and machine learning.

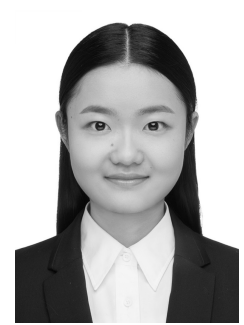

Wenshuo Chen received her BS degree from School of Software, Nanjing University, Jiangsu, China in 2013, and currently is a MS candidate in School of Software in Tsinghua University, Beijing, China. Her research interests include multimedia information retrieval, human action recognition and video event detection.

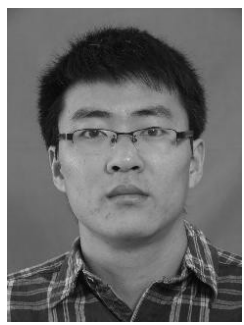

Sicheng Zhao received the Ph.D. degree from Harbin Institute of Technology in 2016. He is now a postdoctoral research fellow in the School of Software, Tsinghua University, China. His research interests include affective computing, social media analysis and multimedia information retrieval.

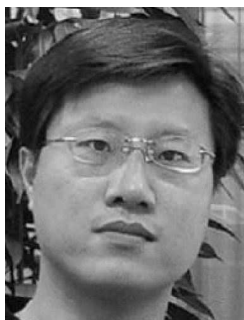

Jungong Han is a tenured faculty member with the School of Computing and Communications at Lancaster University, Lancaster, UK. Previously, he was a faculty member with the Department of Computer and Information Sciences at Northumbria University, UK. His research interests include computer vision, image processing, machine learning, and artificial intelligence.

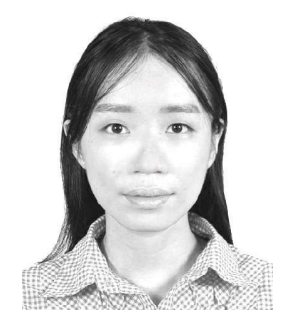

Qiaoyan Liu received her BS degree from School of Software, Beijing University of Posts and Telecommunications, China in 2016, and currently is a MS candidate in School of Software in Tsinghua University, Beijing, China. Her research interests include human face recognition, object tracking and multimedia information retrieval. 